\title{
Uncertainty-based grade modelling of kimberlite: a case study of the Jay kimberlite pipe, EKATI Diamond Mine, Canada
}

\author{
S. Harrison ${ }^{1}$, O. Leuangthong ${ }^{2}$, and B. Crawford ${ }^{1}$ \\ ${ }^{1}$ BHP Billiton Diamonds Inc., Kelowna, Canada \\ ${ }^{2}$ University of Alberta, Edmonton, Canada
}

\section{Introduction}

Understanding uncertainty in resource models is a significant requirement for ore deposit evaluation. Geostatistical simulation is one method that can be used to quantify uncertainty and Sequential Gaussian Simulation is one of the easiest techniques to understand and implement.

The Jay kimberlite pipe is located in the southeastern quadrant of the EKATI property, $5 \mathrm{~km}$ northeast of Misery. Drilling to date has identified three kimberlitic domains characterized by varying lithological properties, including geologic descriptions, diamond grade, dry bulk density, and moisture content. These domains are not separated by hard contacts, but rather by boundaries that are transitional. Within these domains, strong vertical trends exist; in particular, diamond grade increases with depth. For these reasons, Jay required a unique uncertainty-based grade modelling method.

\section{Data}

The Jay kimberlite pipe was first identified as a conductive feature on an airborne electromagnetic survey in 1992. A core hole collared in 1993 confirmed the anomaly as kimberlite and following this, Jay was delineated by eleven additional core holes. Seventeen reverse circulation (RC) drill holes have been drilled to date, providing 223 grade samples. The methods used for RC drilling and grade calculation are outlined in Dyck et al., 2004. All work has been carried out in carats per cubic meter $\left(\mathrm{cpm}^{3}\right)$.

Core and RC holes have been macroscopically logged in detail with microscopic inspection where required. Two core holes have been sampled for thin section analyses to confirm petrographic classifications.

\section{Geology}

The Jay pipe is one of the largest known kimberlite bodies on the EKATI property, modelled to be $11 \mathrm{Ha}$ near surface and roughly circular in shape. The morphology is similar to most other EKATI kimberlites in that it is a steep-sided diatreme that tapers with depth.
From geological logging of core holes and RC samples, three internal geological domains have been interpreted: resedimented volcaniclastic kimberlite (RVK); transitional kimberlite (TransK); and primary volcaniclastic kimberlite (PVK). Boundaries between these sub-horizontal domains are transitional in nature (Figure 1).

Figure 1: A cut-away 3-dimensional isometric view of the Jay kimberlite internal geology showing drilling to date.

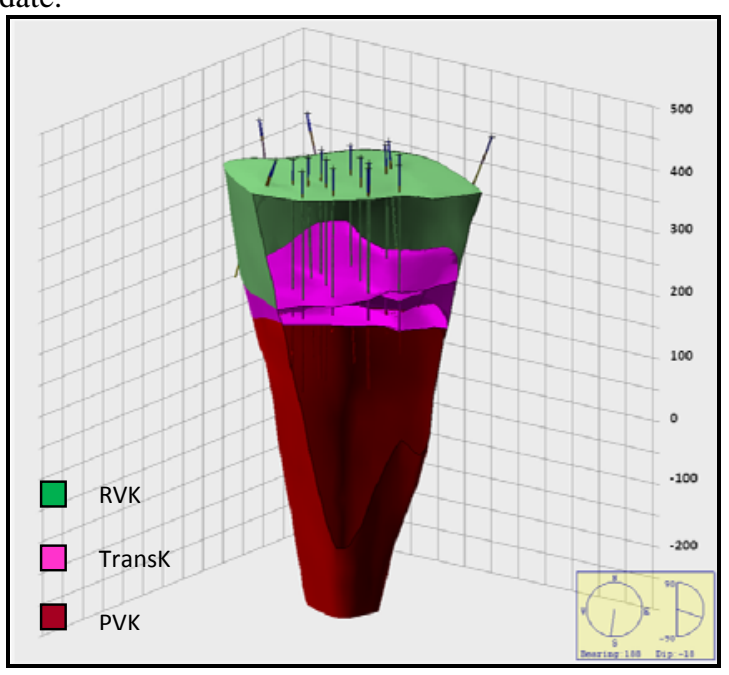

The upper-most domain of the Jay pipe is in-filled with 150 to 175 m of muddy to silty locally varying RVK, primarily defined by olivine size and content and lithic composition and abundance.

Underlying the RVK domain, exists a 25 to $85 \mathrm{~m}$ package of interbedded RVK and PVK of varying degrees of alteration; this unit has been named transitional kimberlite (TransK). Interbeds are typically less than $2 \mathrm{~m}$ and can be as small as $30 \mathrm{~cm}$.

Underlying the TransK domain, the pipe is infilled with massive highly competent medium- to coarse-grained olivine-rich PVK. The upper contact of the PVK domain is marked by the disappearance of RVK and intensely altered PVK. Upon microscopic inspection, mud is absent from the matrix and juvenile clasts are present, typically cored by pseudomorphed olivine. Small, irregularly shaped mudstone and granitic xenoliths are present, but decrease in abundance with depth (Hetman, 2008). 


\section{Grade Modelling}

The current method to achieve uncertainty-based results at EKATI is Sequential Gaussian Simulation (SGS) (Isaaks, 1990). SGS is a combination of simple kriging and Monte Carlo simulation; the former is used to determine the local distribution of uncertainty while the latter is used to randomly draw a simulated value from this distribution. Multiple equally probable realizations are generated by drawing different random numbers. From these realizations, range analyses can be carried out from a block-by-block scale to a global level (Goovaerts, 1997).

While the premise of this technique ensures reproduction of spatial data, it is founded on a very strong assumption of stationarity - in particular, the mean is assumed to be the same at every location in the field (Journel, 1994). However, geological bodies, including kimberlites, often exhibit trends, which are a direct violation of stationarity.

The steps involved in completing uncertainty-based modelling using SGS are as follows: (a) Exploratory Data Analyses (EDA) investigating the nature of internal domains, contacts, trends, and spatial distributions; (b) data transformation to normal space either using a normal scores transform or the stepwise conditional transform (Leuangthong, 2003); (c) variography; (d) simulation; (e) validation and backtransformation to original units; and (f) postprocessing, including up-scaling to different grid sizes and calculating the uncertainty around the estimate.

\section{Exploratory Data Analyses}

As the Jay kimberlite contains three distinct geological domains, it is important to understand their relationship to grade. Various EDA methods, including histograms, scatter-plots, and contact analyses, prove that grade is transitional and that the interpreted geological domains are not applicable. These methods also show that there is a clear trend of increasing grade with depth. It is important to consider this trend during simulation due to the small sample population, but also its magnitude - grade varies by more than a factor of two over the given elevation (Figure 2).

\section{Trend Modelling}

A variety of methods are available to model trends, including hand mapping, moving window averages, inverse distance, simple kriging, ordinary kriging, and universal kriging (Leuangthong and Deutsch, 2004; McLennan, 2007). Trend models should represent large scale features of the data; however, it is easy to over-fit the trend which may lead to an overconstrained uncertainty model from simulation.

A vertical polynomial linear trend was modelled and the equation of this line was used to create a gridded model. Trend values were extracted from the gridded model at the sample locations. Figure 2 illustrates the trend model overlaid on a scatter-plot of grade versus elevation.

Figure 2: The polynomial linear trend model plotted on a grade scatter-plot with a $45 \mathrm{~m}$ moving window average. This also shows the continuous nature of the grade variable.

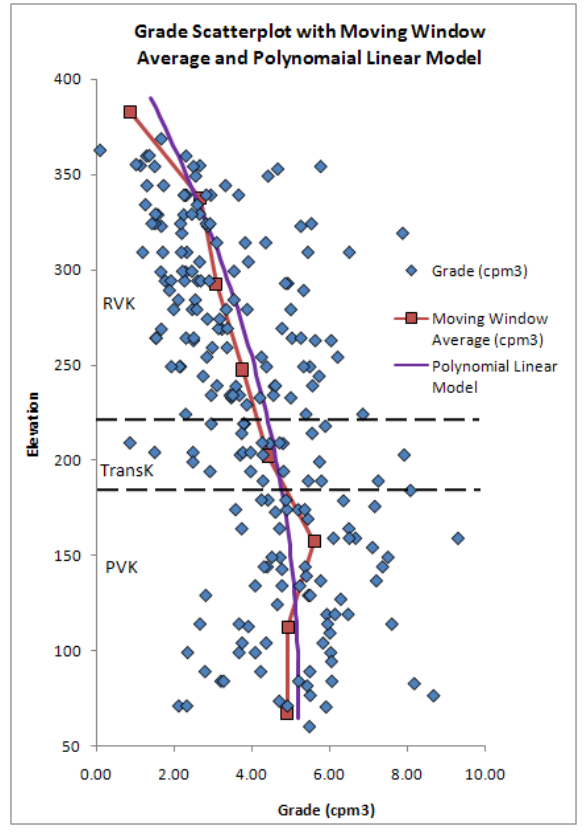

Incorporating the Trend into Simulation

Decomposing the original variable into a trend and residual component is a common approach to working with trends (Deutsch, 2002). However, this method may lead to negative values since the relationship between the trend and residual is ignored (Leuangthong \& Deutsch, 2004). To avoid this possibility, the stepwise conditional transform (SCT) was used.

Variograms were calculated from the SCT grade data set for use in simulation and multiple equally probable SGS realizations were created. The results were verified and back-transformed to provide a spatial estimate of grade over the entire pipe (Figure 3).

The statistical and spatial distribution of the resulting realizations reproduced that of the original grade data, proving that the results are valid and robust (Figure 4).

\section{Uncertainty Quantification}

Uncertainty can be quantified on a variety of scales - it is important to understand the scale of the calculation and the results. At EKATI, results are reported on two scales: a global scale and a bench or level scale. To calculate global grade ranges, the mean grade of each realization is calculated. The realizations are ranked and assigned a probability, and uncertainty ranges are calculated at given confidence intervals. 
To calculate bench grade ranges, each bench of each realization is averaged. Again, for each bench, the realizations are ranked and uncertainty ranges are calculated at given confidence intervals. This method can be applied to any scale required.

Figure 3: A cross-section of one back-transformed realization of the Jay kimberlite.

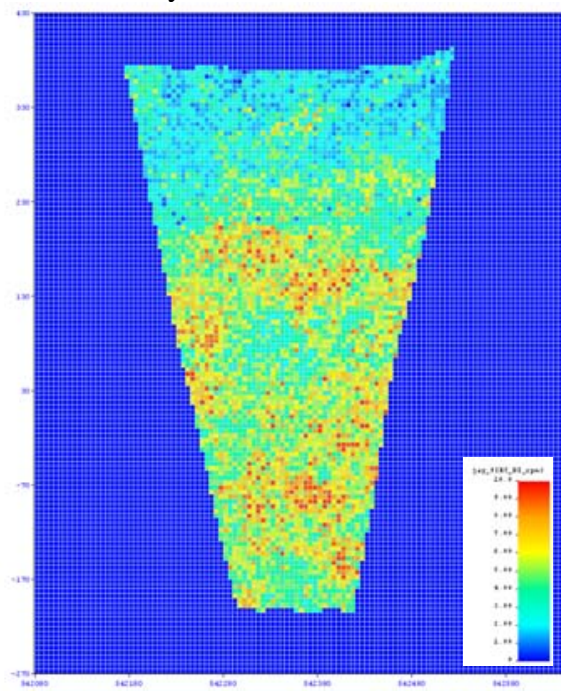

Figure 4: Moving window averages $(45 \mathrm{~m})$ of five realizations plotted on a grade scatter-plot with the moving window average of the grade data, and the trend model.

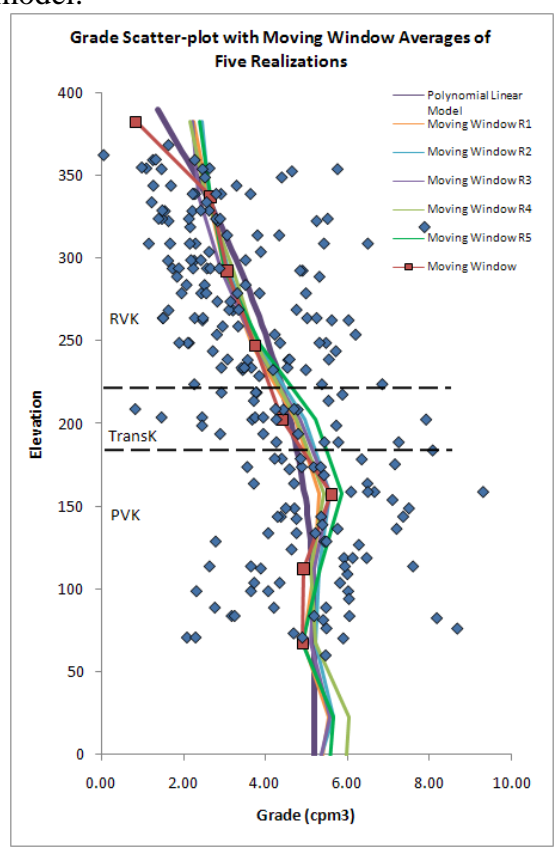

\section{Discussion}

The validity of the resource model requires a clear understanding of the geology of the deposit. As the correlation between different variables and geology can vary, it is important to understand these relationships before embarking on a resource modelling exercise. The Jay kimberlite's transitional internal boundaries and distinct trend are prime examples of how this understanding can impact the modelling method chosen and therefore, the results.

Trend modelling is important to many resource studies. The general idea of the trend is to capture large-scale changes in the data; however, there are no explicit guidelines nor generally accepted approach to trend modelling. Acceptance and integration of a trend model is highly dependent on the modeller, yet it can have a large impact on the final simulated model. For this reason, multiple trend models should be considered.

The uncertainty-based diamond grade model presented in this study is consistent with standard geostatistical modelling practices. Sensitivity analyses were performed to ensure that reasonable parameters were used and that the results reproduced the statistical and spatial distribution of the original data. The presence of a trend required further consideration during simulation to ensure that it was properly reproduced. It was particularly important to ensure that the resulting grade ranges from simulation were not too narrow. If this was the case, the trend model is likely over fit and has consequently constrained the simulated results.

The use of SCT with SGS is relatively easy to implement. It has wide-ranging applications for kimberlite deposits based on their trends due to emplacement processes and, for grade in particular, their small sample populations due to the large sample size required and associated high cost.

\section{References}

Deutsch, C.V., 2002. Geostatistical Reservoir Modeling, Oxford University Press, New York.

Dyck, D.R., Oshust, P.O., Carlson, J.A., Nowicki, T.E., Mullins, M.P., 2004. Effective resource estimates for primary diamond deposits from the EKATI Diamond Mine $^{\mathrm{TM}}$, Canada In: Mitchell, R.H., Grütter, H.S., Heaman, L.M., Scott Smith, B.H., Satchel, T. (Eds.), 8th International Kimberlite Conference Selected Papers, Vol. 1, 317 - 335.

Goovaerts, P., 1997. Geostatistics for Natural Resources Evaluation. Oxford University Press, New York, 483.

Hetman, C.M., 2008. Brief Petrography of Samples from the Jay Kimberlite, EKATI Mine, NWT, Canada, internal report, MSC08, work in progress.

Isaaks, E.H., 1990. The application of Monte Carlo methods to the analysis of spatially correlated data. PhD Thesis, Stanford University, Stanford, CA.

Leuangthong, O., 2003. Stepwise conditional transformation for multivariate geostatistical simulation. PhD Thesis, University of Alberta, Edmonton, AB.

Leuangthong, O., Deutsch, C.V., 2004. Transformation of residuals to avoid artifacts in geostatistical modelling with a trend. Mathematical Geology, Vol. 36, No. 3, 287 $-305$.

Journel, A.G., 1994. Modeling uncertainty: some conceptual thoughts. Geostatistics for the next Century, Kluwer, 30 -43 .

McLennan, J., 2007. The decision of stationarity. PhD Thesis, University of Alberta, Edmonton, AB. 